\title{
A novel low complexity high efficiency hybrid PAPR reduction for OFDM systems
}

\begin{abstract}
In this paper, we propose a novel hybrid multiplicative-additive technique to reduce the peakto-average power ratio (PAPR) in orthogonal frequency division multiplexing (OFDM) systems. This technique consists of two inverse fast Fourier transform (IFFT) blocks. The input symbols of the first IFFT are the mapped symbols, whereas the input symbols of the second IFFT are the summations of the absolute value of the real part of the outer signal constellation points and zeros symbols. First, the output of the two IFFT blocks is partitioned into four subblocks, which are subsequently used to rearrange the subblocks with padding zeros in a specific manner. Then, a new optimization scheme is introduced, in which only a single two-phase sequence and four iterations needs to be applied. Numerical analysis shows that the hybrid proposed technique achieves better bit error rate (BER) and PAPR reduction performance than partial transmit sequences (PTS) multiplicative technique and tone reservation (TR) additive PAPR technique. The other salient feature of this scheme is that no side information (SI) is needed which increases transmission efficiency.
\end{abstract}

Keyword: OFDM; PAPR; PTS; TR 